\title{
72 The biomechanics of running
}

Barefoot or minimally shod runners tend to land on the fore- or mid-foot. In contrast, the running shoes popular today, with their elevated and cushioned heels, tend to cause many joggers to rear-foot strike, thereby exposing them to a higher incidence of repetitive stress injuries (Lieberman et al. 2010). As a result of this cushioning, the collision forces exerted on the forefoot are lower than on the heel.

The basic skeletal structure of the foot is made up of 26 bones, with a total of 45 muscles per leg necessary to carry the body and ensure the body's locomotion in its upright position.

During locomotion, the feet must absorb two force peaks per step: a shock-absorbing impact peak approximately 20-30 milliseconds (ms) after ground contact and a push-off peak after a further $70 \mathrm{~ms}$ that again propels the foot in the direction of walking. In the push-off phase, the bone and joint stresses are higher than during the foot-strike phase. During a normal landing phase, the foot initially contacts the ground with its front outer edge, then rolls inwardly and pronates during a very short stance phase.

The stresses exerted on the lower extremities during this phase are tremendous. Depending on the speed of locomotion, each step causes the ankle joint to be subject to 2.2 to 4.8 times the body's weight for 20-40 ms. On average, the weight-bearing stresses for a person weighing $75 \mathrm{~kg}$ running a distance of
1 kilometer (1200 steps) add up to around 160 tons spread over 18 seconds for each foot.

In other words, those individuals who, in addition to their daily walking distances, do $20 \mathrm{~km}$ per week of jogging over a period of 40 years will have subjected each ankle to about 6.5 million tons of stress spread over about 200 hours. This load-withstanding feat demonstrates the amazing adaptability of biological tissues. The training effect on muscle growth, in particular, is measurable just a few weeks after beginning an exercise regimen or increasing the scope of training activities ( $\triangleright$ Chapter 79 ). Connective tissue, however, does not behave quite as effectively during these adaptation processes. Because of their lower metabolic activity, structures such as cartilage, tendons, ligaments and capsules need up to 12 months to adapt.

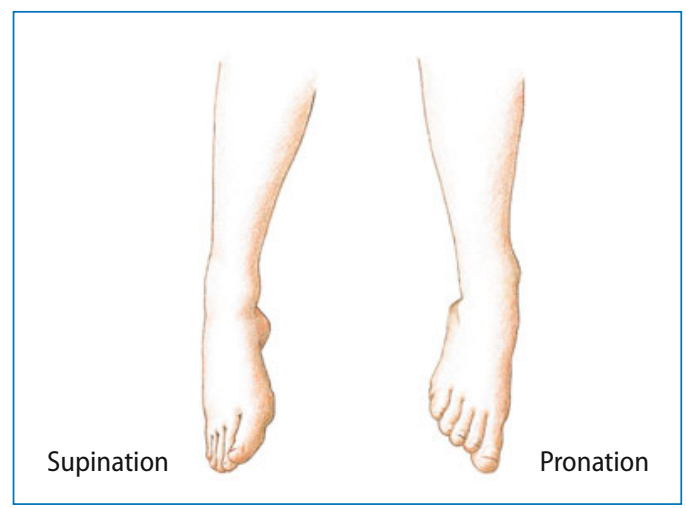

- Fig. 72.1 Load-bearing foot postures (exaggerated representation) 